\title{
Coagulation measurement from whole blood using vibrating optical fiber in a disposable cartridge
}

\author{
Yusuf Samet Yaraş \\ Ali Bars Gündüz \\ Gökhan Sağlam \\ Selim Ölçer \\ Fehmi Civitçi \\ İbrahim Baris \\ Göksenin Yaralioğlu \\ Hakan Urey
}




\title{
Coagulation measurement from whole blood using vibrating optical fiber in a disposable cartridge
}

\author{
Yusuf Samet Yaraş, ${ }^{a}$ Ali Bars Gündüz, ${ }^{a}$ Gökhan Sağlam, ${ }^{a}$ Selim Ölçer, ${ }^{a}$ Fehmi Civitçi, ${ }^{b}$ ibrahim Baris, ${ }^{c}$ \\ Göksenin Yaralioğlu, ${ }^{d}$ and Hakan Urey ${ }^{a, *}$ \\ ${ }^{a}$ Koc University, College of Engineering, Department of Electrical and Electronics Engineering, Istanbul, Turkey \\ bIstanbul Technical University, Department of Electronics and Communication Engineering, Faculty of Electrical and Electronics Engineering, \\ Istanbul, Turkey \\ ${ }^{c}$ Koc University, College of Sciences, Department of Molecular Biology and Genetics, Istanbul, Turkey \\ ${ }^{\mathrm{d} O z y e g i n}$ University, Faculty of Engineering, Department of Electrical and Electronics Engineering, Istanbul, Turkey
}

\begin{abstract}
In clinics, blood coagulation time measurements are performed using mechanical measurements with blood plasma. Such measurements are challenging to do in a lab-on-a-chip (LOC) system using a small volume of whole blood. Existing LoC systems use indirect measurement principles employing optical or electrochemical methods. We developed an LoC system using mechanical measurements with a small volume of whole blood without requiring sample preparation. The measurement is performed in a microfluidic channel where two fibers are placed inline with a small gap in between. The first fiber operates near its mechanical resonance using remote magnetic actuation and immersed in the sample. The second fiber is a pick-up fiber acting as an optical sensor. The microfluidic channel is engineered innovatively such that the blood does not block the gap between the vibrating fiber and the pick-up fiber, resulting in high signal-to-noise ratio optical output. The control plasma test results matched well with the plasma manufacturer's datasheet. Activated-partial-thromboplastin-time tests were successfully performed also with human whole blood samples, and the method is proven to be effective. Simplicity of the cartridge design and cost of readily available materials enable a low-cost point-of-care device for blood coagulation measurements. () The Authors. Published by SPIE under a Creative Commons Attribution 3.0 Unported License. Distribution or reproduction of this work in whole or in part requires full attribution of the original publication, including its DOI. [DOI: 10.1117/1. JBO.22.11.117001]
\end{abstract}

Keywords: fiber optic sensor; blood coagulation; activated partial thromboplastin time; point-of-care; lab-on-a-chip.

Paper 170158R received Mar. 15, 2017; accepted for publication Oct. 12, 2017; published online Nov. 10, 2017.

\section{Introduction}

Blood coagulation tests are required for most surgical operations, ${ }^{1}$ hepatic and renal disorders, ${ }^{2}$ and dengue hemorrhagic fever conditions. ${ }^{3}$ Furthermore, patients receiving anticoagulant treatments ${ }^{4}$ and patients with a risk of cardiac problems such as embolism, atrial fibrillation, or stroke need to undergo blood coagulation tests periodically since the dosage of anticoagulants must be adjusted according to blood coagulation test results. ${ }^{5}$

Over 800 million coagulation tests are performed each year, mostly in hospitals and clinical laboratories for emergency situations and surgical operations, as well as periodic monitoring of certain patients. ${ }^{6}$ There are two golden standard techniques extensively used in clinical coagulation monitoring: optical and direct mechanical. ${ }^{7}$ All clinical measurements of coagulation monitoring are conducted on plasma samples extracted from whole blood by centrifuging.

Portable point-of-care (PoC) coagulation monitoring devices have also been developed for emergency situations and for patient self-testing. ${ }^{7,8}$ Such coagulation devices need to operate with a small volume (10 to $20 \mu \mathrm{L}$ finger prick sample) and without plasma extraction. ${ }^{7,9}$ Existing PoC coagulation measurement devices primarily use optical or electrochemical measurement principles, which are indirect methods and have limitations. ${ }^{8}$ Electrochemical detection method is the dominant method

*Address all correspondence to: Hakan Urey, E-mail: hurey@ku.edu.tr used in commercial PoC coagulation measurement devices e.g., CoaguChek XS (Roche Diagnostics) ${ }^{7}$ and i-STAT (Abbott Diagnostics). ${ }^{5}$ As blood coagulates, electrical resistance of the blood changes and gives an indication of coagulation time. ${ }^{10}$ These devices can operate with small sample volumes using cartridges or strips. ${ }^{11}$ However, they have two major disadvantages related to reliability of the results: (i) chemical and biological coatings applied in the cartridges need to be consistent for all cartridges since an induced electrical current strongly depends on the ionic composition of the blood, ${ }^{7}$ (ii) results are susceptible to multiple drug interactions and certain health conditions. ${ }^{12,13}$ Although the coating consistency problem can be solved with a well-controlled strip fabrication process and strip calibration, the second problem cannot be addressed easily.

In optical transmission-based methods, the signal-to-noise ratio (SNR) of the monitored signal is low due to the complex nature of whole blood. ${ }^{14,15}$ On the other hand, microfluidic constriction-based optical detection methods utilize flow rate change of blood in a microfluidic channel by clot formation. ${ }^{7}$ The readout is usually performed with simple optical sensors. However, microfluidic cessation-based devices are very sensitive to minute viscosity variations among individual users and thus require calibration, which is not a straightforward process for every patient. ${ }^{16,17}$ Another technique is measurement of clot formation by inspecting fibrins visually: a wheel-like structure tries to catch fibrins formed during coagulation and a photodetector detects fibrins. ${ }^{18}$ However, the size of the device and the 
complex blood insertion mechanism degrade the suitability of this system as a PoC device. Previously, dynamic light scattering-based methods are also proposed. ${ }^{19,20}$ However, these systems have very complex measurement platforms (blood circulation path and detector array arrangements), which are not compatible with PoC applications.

An MEMS-based coagulation measurement platform, which is able to perform direct mechanical measurement by employing a resonating MEMS cantilever, has been developed for viscosity and plasma coagulation measurements by our group. ${ }^{21-25}$ Although results with plasma were very promising, optomechanical tolerances were tight and whole blood results were not reliable due to scattering and low optical transmission.

In this study, we developed a portable lab-on-a-chip (LoC) system for direct measurement of coagulation time using whole blood. The system consists of a reader unit that houses the electronics, light source, and photodetectors and a disposable cartridge inserted in the reader unit. Our method closely mimics the golden standard used in clinics and overcomes the challenges associated with small sample volumes, strict optomechanical alignment requirements, and whole blood operation. Our method is a direct mechanical method as it uses a vibrating optical fiber operated in the blood sample. Low SNR problems were addressed using an engineered microfluidic channel, which separates blood interaction and measurement regions of the vibrating fiber.

\section{Materials and Methods}

\subsection{Cartridge and Reader Unit}

The schematic top view of the measurement setup is shown in Fig. 1(a). The cartridge is a single-use disposable piece with fluidic inlet and outlets for whole blood. The measurement is performed in a microfluidic channel where a vibrating fiber and a pick-up fiber are placed inline with a small gap in between. The vibrating fiber operates near its mechanical resonance using magnetic actuation and immersed in the whole blood sample. The magnetic actuation is achieved by a small nickel piece attached to the free-end of the vibrating fiber. The pick-up fiber is immobilized in the channel and collects the optical signal from the vibrating fiber. A key feature of the system is that the microfluidic channel has a fluid stop region, which prevents the blood from getting into the gap between the vibrating fiber and the pick-up fiber. Having a clear optical path in the fluid stop region avoids scattering and optical losses, which results in high SNR optical output. Furthermore, no electrical connection to the cartridge is needed since both the actuation and the readout are done remotely.

The electronics, optical components, and electrocoil surrounding the disposable cartridge are part of the reader unit. The system uses an electromagnetic actuator to drive a vibrating optical fiber inside the cartridge and an optical sensor. The disposable cartridge is placed on top of an electromagnetic coil embedded inside an aluminum fixture. The optical readout employs a light-emitting diode (LED) and a photodetector, which are pigtailed to multimode fibers in the reader unit. These multimode fibers are butt coupled to two respective fibers that are placed in the cartridge as detailed in the next section. The optical signal generated in the cartridge is processed by the postdetection electronics.

Control electronics consist of a signal generator, a current amplifier to drive the electromagnetic coil, and a lock-in amplifier (Zurich Instruments HF2LI) to detect the amplitude and the relative phase of the photodetector signal. The signal generator operates at a constant frequency near the mechanical resonance of the vibrating optical fiber.

A photo of the measurement setup with cartridge is shown in Fig. 1(b).

Precise temperature control is very important because coagulation time heavily depends on temperature. ${ }^{26} \mathrm{~A}$ custom temperature control unit was designed for reliable and repeatable testing. The temperature control unit consists of heating wire buried inside the aluminum fixture, a temperature sensor (Analog Devices AD590), and a custom design controller circuit. Moreover, the aluminum fixture, cartridges, and fluid samples were preheated to $37^{\circ} \mathrm{C}$. Temperature stability of $\pm 0.05^{\circ} \mathrm{C}$ at $37^{\circ} \mathrm{C}$ was achieved with the described setup.

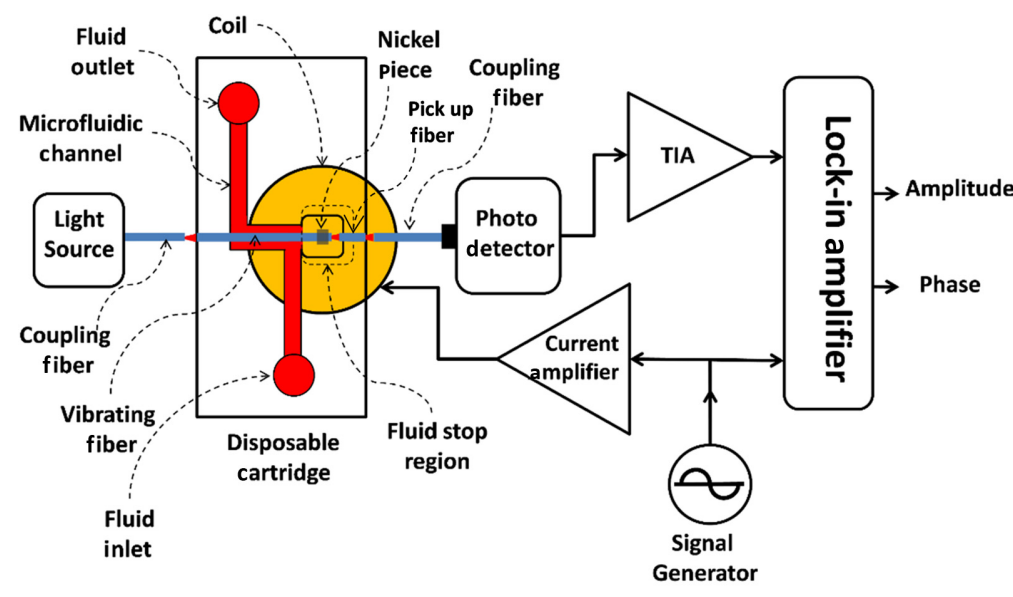

(a)

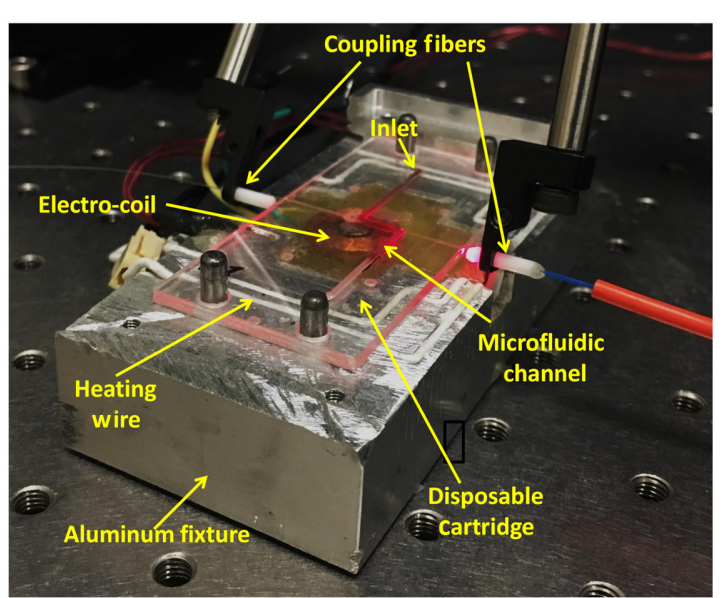

(b)

Fig. 1 (a) Schematic of the measurement setup. Optical fiber is actuated remotely by an electrocoil. Optical signal is collected through a silicon photodetector. Amplitude and phase of the signal are monitored by a lock-in amplifier. (b) A photo of the measurement setup with cartridge. Blood sample is inserted into the microfluidic channel through an inlet. Blood flows with capillary motion into the cartridge. 


\subsection{Measurement Principle}

When blood or plasma coagulates, a sudden change in viscoelasticity occurs by clot formation. ${ }^{27}$ There are two elements of viscoelasticity: viscosity and elasticity. The viscosity of the liquid affects the resonant frequency and quality factor of the vibrating fiber. As the viscosity of the liquid medium changes, resonant characteristics of the fiber change due to hydrodynamic loading, as detailed in Sader's study. ${ }^{28,29}$ When blood coagulates, the viscosity increases and causes a decrease in the resonant frequency and quality factor of the cantilever. Furthermore, elasticity of the blood increases by fibrin formation; hence, the spring constant of the fiber cantilever increases, ${ }^{30}$ which reduces the vibration amplitude and increases the resonance frequency. The implemented optical readout principle is based on modulating the optical power coupled from the vibrating fiber to the pick-up fiber. There is a net change in system dynamics, which produces a clear optical signal when the amplitude and the phase of the optical detector are monitored.

\subsection{Cartridge Fabrication}

The cartridge has two parts: body and optical fibers. We used multimode optical fibers in the cartridge with a core diameter of $400 \mu \mathrm{m}$ and cladding diameter of $440 \mu \mathrm{m}$. For both the resonating and pick-up fibers, jackets were stripped and cut to the designed length with a fiber optic cleaver. For a better light coupling, both ends of the fibers were polished using a fiber polishing kit and were cleaned with isopropyl alcohol and distilled water. A high purity nickel wire piece with a length of $1 \mathrm{~mm}$ and a diameter of $350 \mu \mathrm{m}$ was glued onto the resonating fiber part using a low viscosity super glue (Pattex Sekundenkleber Plastix Flüssig, Germany). The glued nickel part makes magnetic actuation possible for the resonating fiber.

The body of the cartridge consists of two polymethyl methacrylate (PMMA) parts. Microfluidics and grooves are CNC machined into PMMA sheets with a high accuracy micromilling machine (MDA Presicion). Grooves are designed to hold the fibers tightly. Precise machining of these grooves is important because of the alignment of the fibers with respect to each other. The machining process was calibrated vigorously on a regular basis; test samples were measured with a white light interferometer. An accuracy of $\pm 5 \mu \mathrm{m}$ was achieved in microfluidic channels. There are one inlet, one outlet, a sensor chamber, and a fluid stop region as microfluidics. The microfluidic channel and inlet geometries are specifically designed to provide fluid flow without pumping or surface functionalization. Thus, eliminating extra cost and complexity of pumping and surface functionalization enables a more robust and low cost cartridge.

Employing optical readout in blood is challenging because of the high absorbent and scattering characteristics of red blood cells. ${ }^{31}$ Thus, we developed a technique that enables optical readout using a fluid stop region. The fluid stop region is located where two ends of the fiber parts meet each other. This region prevents fluid from going in between the two fiber ends via capillary action. Capillary action is the tendency of fluid to flow into smaller gaps rather than larger gaps. The fluid stop region is machined much larger in terms of both height and width with respect to the sensor chamber to decrease the capillary force inside this region. The adhesion of the liquid to channel walls and cohesion of the liquid around air-fluid interface prevent fluid from going into the fluid stop region, where the two fibers face each other. Furthermore, trapped air in the fluid stop aids prevention of leakage of blood into the fluid stop region. Figure 2(a) shows the fluid stop region, sensor chamber, fibers and blood flow with LED source, photodetector, and coil. In our cartridges, the sensor chamber is machined to $800-\mu \mathrm{m}$ depth and 1-mm width whereas the fluid stop region is machined to 1.6-mm height and 2-mm width. Since blood flow inside the cartridge is also promoted by capillary motion, a possible forced flow into the fluid stop region is prevented as shown in Fig. 2(b). Preventing light from going through blood by employing a fluid stop region enables a robust optical readout scheme with high SNR. Note that the Ni piece is not attached to the fiber cantilever shown in Fig. 2(a), since it is not a functional cartridge but fabricated only for liquid stop region optimization.

The assembly procedure of the cartridge can be explained as follows. Fiber parts are placed into grooves on the bottom cartridge body and prefixed with $1 \%$ aqueous polyvinyl alcohol solution and dried in vacuum chamber for $20 \mathrm{~min}$. Then, two body parts of the cartridge are tightly put together with clamps. UV-cured epoxy (Norland Products Optical Adhesive 81) is wicked between two body parts and cured with UV light. Since wicking epoxy automatically stops at the edges of microfluidic channels due to capillary action, this bonding method

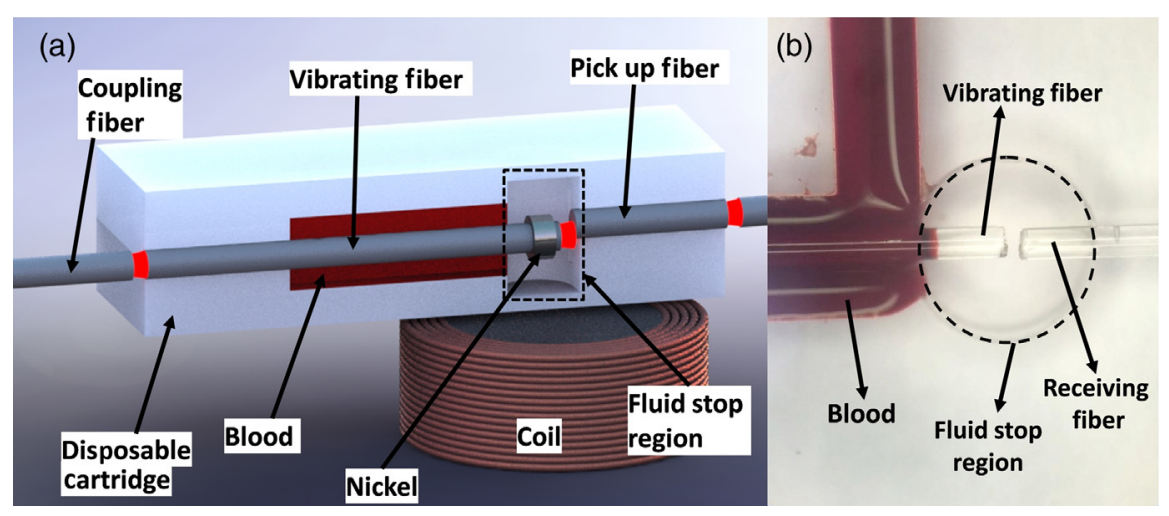

Fig. 2 (a) A cross sectional 3-D sketch of the cartridge with electrocoil. Blood inlet and outlet are not shown. Blood does not get into the fluid stop region due to capillary force at the boundary. (b) Top view microscope picture of the fluid stop region with blood in the microfluidic channel. Note that the tip of the fiber is free to move; there is no physical barrier. Also note that the Ni piece is not attached to the fiber cantilever shown here since it is not a functional cartridge but fabricated only for fluid stop illustration. 
enables easy all around sealing for complex channel geometries. Note that clean room processes are not needed for the sensor fabrication, which reduces cost and time significantly.

\section{Results and Discussion}

\subsection{Sensor Characterization}

The system was first characterized with various samples. Frequency sweeps were conducted with the same cartridge in air, distilled water, uncoagulated plasma, and coagulated plasma. Figures 3(a) and 3(b) show the frequency response of the system for different fluids. The resonant frequency of the sensor in air is $4703 \mathrm{~Hz}$, and the quality factor is 62 . When distilled water is inserted into the microfluidic channel, the resonance frequency drops to $4300 \mathrm{~Hz}$ and the quality factor drops to 34 due to hydrodynamic effects. Frequency sweeps were performed before and 3 min after coagulation with control plasmas. Similar to the air and distilled water tests, both the resonance frequency and the quality factor decreased. In uncoagulated plasma, the resonance frequency was $4214 \mathrm{~Hz}$, and the quality factor was 29; after coagulation, the resonance frequency and the quality factor dropped to $4191 \mathrm{~Hz}$ and 22, respectively. The change in resonance frequency is not very large, but the changes in amplitude and phase response of the system are significant. As can be seen in Fig. 3(b), the amounts of change for amplitude and phase are different for particular frequencies. For instance, a decrease in amplitude is maximum at around $4200 \mathrm{~Hz}$ whereas a change in phase is maximum at around 4120 and $4350 \mathrm{~Hz}$. Moreover, the phase can decrease or increase depending on the drive frequency whereas amplitude only decreases due to increased viscous drag and elasticity. Since the proposed system is more sensitive for amplitude, measurements were conducted at a fixed frequency around the resonant frequency of the vibrating fiber. The described characterization procedure was applied to all cartridges to minimize the effect of differences between individual cartridges and samples to achieve accurate and repeatable results with high SNR.

Blood coagulation times are usually measured with either PT or activated partial thromboplastin time (aPTT) test protocols. ${ }^{32}$ The average PT test time is around $13 \mathrm{~s}$ while the average aPTT test time is much longer at around $30 \mathrm{s.}{ }^{33}$ Even with a severe abnormality, aPTT tests do not exceed 90 s. ${ }^{34}$ Figures 3(c) and 3(d) show long-term amplitude and phase stability of the system in distilled water for $10 \mathrm{~min}$, which is more than six times longer than aPTT times of severely abnormal blood samples. The stability measurement yielded a standard deviation of $1.2 \mathrm{mV}$ and a standard deviation of $0.35 \mathrm{deg}$ in phase difference between the drive and readout signals. SNR of the amplitude is calculated as 125 by taking the standard deviation of $1.2 \mathrm{mV}$ as the noise floor of the system and signal as $150 \mathrm{mV}$, which is the average amplitude change due to coagulation. Similarly, SNR of the phase is calculated as 18.5 by taking the standard deviation of $0.35 \mathrm{deg}$ as the noise floor of the system and signal as $6.5 \mathrm{deg}$, which is the average phase change due to coagulation.

\section{2 aPTT Measurements with Control Plasma}

In the scope of this report, aPTT tests were conducted using the proposed system using control plasma and whole blood samples. We used three different control plasma samples: normal (DIAGEN, UK, lot No.: KP93), abnormal 1 (DIAGEN, UK, lot No.: AB1-80), and abnormal 2 (DIAGEN, UK, lot No.: AB2-79). While normal control plasma samples have coagulation times similar to a healthy person's, the abnormal 1 sample has mild abnormality in coagulation time and the abnormal 2 has severe abnormality. Before measurements, all control samples and reagents were warmed to $37^{\circ} \mathrm{C}$ in a water bath, and the aluminum fixture was preheated to $37^{\circ} \mathrm{C}$ by the temperature control
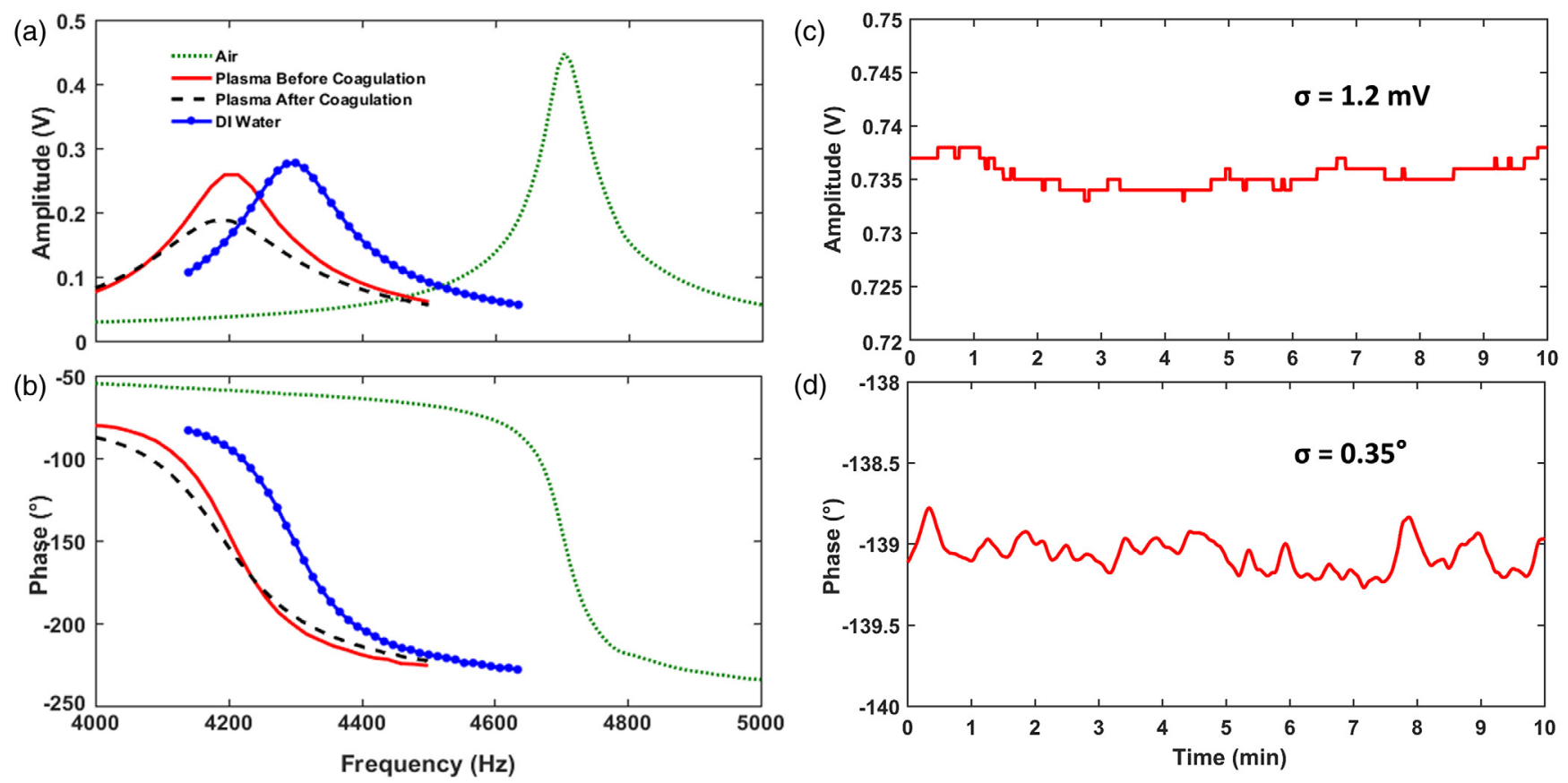

(d)

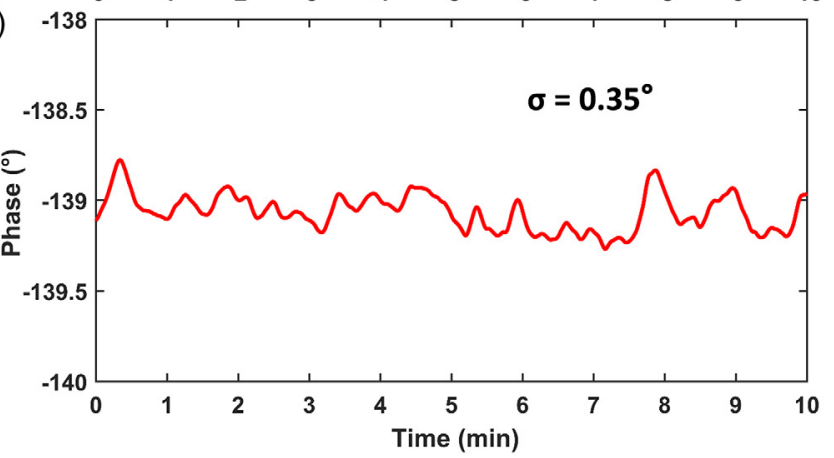

Fig. 3 Frequency response of the system to air, distilled water, uncoagulated blood plasma, and blood plasma after coagulation. Amplitude and phase change are shown in (a) and (b), respectively. (c) Amplitude and (d) phase stability of the sensor in distilled water for 10 min. Amplitude has a standard deviation of $1.2 \mathrm{mV}$, and phase has a standard deviation of $0.35 \mathrm{deg}$. 
unit. For aPTT tests, $50 \mu \mathrm{L}$ of control plasma was mixed with 50- $\mu \mathrm{L}$ micronized silica/platelet substitute mixture (DIAGEN, UK, lot No.: SP66), which is a common aPTT reagent, used in coagulation tests. The control plasma-reagent mixture was incubated for exactly $5 \mathrm{~min}$. After incubation, $50 \mu \mathrm{L}$ of the $25-\mathrm{mM} \mathrm{CaCl}{ }_{2}$ solution was mixed with the control plasmareagent mixture, and $12 \mu \mathrm{L}$ of this final mixture was inserted into the cartridge immediately. Since control plasma samples are citrated, the coagulation process does not start until the introduction of $\mathrm{CaCl}_{2}$. Therefore, mixing the $\mathrm{CaCl}_{2}$ solution into the mixture is taken as $t=0$. While coagulation was observed through amplitude, the remaining sample was pipetted regularly and observed for clot formation to have an external confirmation for coagulation.

The amplitude and phase response of the system for aPTT test from control plasma can be seen in Fig. 4(a). A sudden decrease in both amplitude and phase is observed around $48 \mathrm{~s}$ due to clotting. The drive signal frequency was chosen to maximize the change in the signal amplitude. Therefore, the phase change is less noticeable. The circular cross section shape of the optical fiber has significantly less drag coefficient compared with the flat surface of conventional cantilevers that are used in biosensor applications. A more aerodynamic structure shape results in less drag force applied by the viscous medium. Therefore, a change in the movement of the optical fiber is less sensitive to viscosity change. However, the optical readout scheme presented in this report is very sensitive to mechanical displacement, which results in a high electrical readout amplitude. On the other hand, the sensitivity of phase change is lower than the sensitivity of amplitude change since it is inherently related to the cantilever structure regardless of the readout scheme. Since alignment of the cartridge to the reader unit is less sensitive compared with MEMS-based sensors, ${ }^{22,23}$ the amplitude readout does not suffer from coupling variations among different cartridges. Therefore, we decided to monitor coagulation through amplitude change.

The signal amplitude is observed for more than $1 \mathrm{~min}$ in the system. A polynomial was fitted to amplitude data, and the first derivative of this polynomial was calculated. In Fig. 4(b), raw amplitude data, polynomial fitted data, and first derivative of the polynomial fit are shown. There are two important points in the derivative data. First, the derivative that is zero can be interpreted as the start of coagulation. Second, the local minima of the derivative can be interpreted as the midpoint of the coagulation. Since the local minima well overlaps with both plasma manufacturer data sheet and control samples that were observed outside the channel by pipetting to test the coagulation, the local minima time of the first derivate was used for quantitative coagulation times. Figure 5 shows measurement results and their derivative for normal, abnormal 1 , abnormal 2 control plasma, and whole blood samples. The measurement of each control plasma sample was repeated three times for different plasma samples to check the reproducibility. Cartridge to cartridge variation caused by the fabrication process and variation among control plasma samples may affect the overall system response. Therefore, it is important to have reproducible data with different cartridges and samples to assure a reliable diagnostic assay.

Figure 5(a) shows normalized aPTT test results obtained from different control plasma samples. Since every coagulometer platform has a different sensing mechanism and different test protocols, there is a high chance of obtaining different results from the same sample. To prevent variations among different platforms, normalized aPTT values are used. ${ }^{35}$ Normalized aPTT is calculated by dividing the test result by the average aPTT time for the specific setup and protocol. First derivatives of the normalized amplitudes are used for time calculations as explained earlier [Fig. 5(b)]. The average of results obtained from normal control plasma samples were equated to mean a normalized aPTT value of 1.03 , which is given in the plasma manufacturer datasheet. For abnormal 1 and abnormal 2 normalized aPTT values, 1.03 was used as reference. Unlike the phase readout, the amplitude level may change cartridge to cartridge due to light coupling condition variances and the fabrication process. Therefore, in addition to aPTT time normalization, amplitude levels were also normalized to unity by dividing maximum amplitude for convenient data representation. The normalized aPTT ratio, standard deviation $(\sigma)$, and relative standard deviation (RSD) for control plasma samples are shown in Table 1. Test results match well with data provided by the manufacturer datasheet. There is $1.36 \%$ RSD for abnormal 1 and $0.9 \%$ for abnormal tests.

\section{3 aPTT Measurements with Whole Blood}

While clinics perform coagulation measurement using large volumes of blood and separate the plasma, PoC devices perform measurements from low volumes without plasma separation.
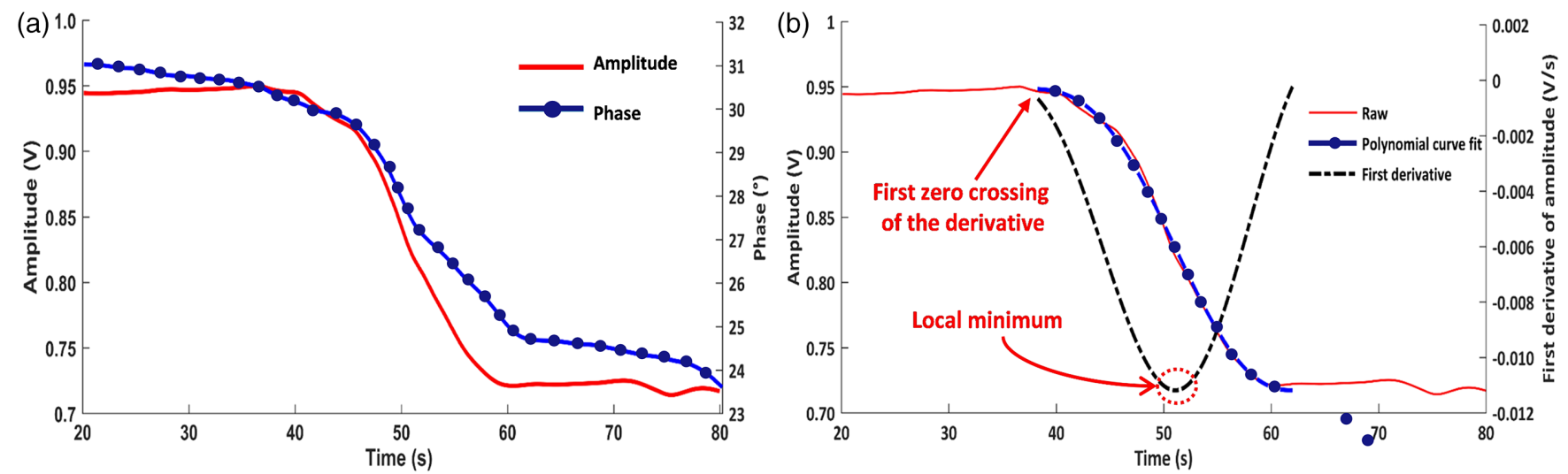

Fig. 4 (a) Amplitude and phase change during coagulation of abnormal 1 control plasma. (b) Polynomial fit to amplitude and its first derivative. Time of local minimum of the derivative is reported as coagulation times. First zero crossing of the derivative indicates the clot formation time. 

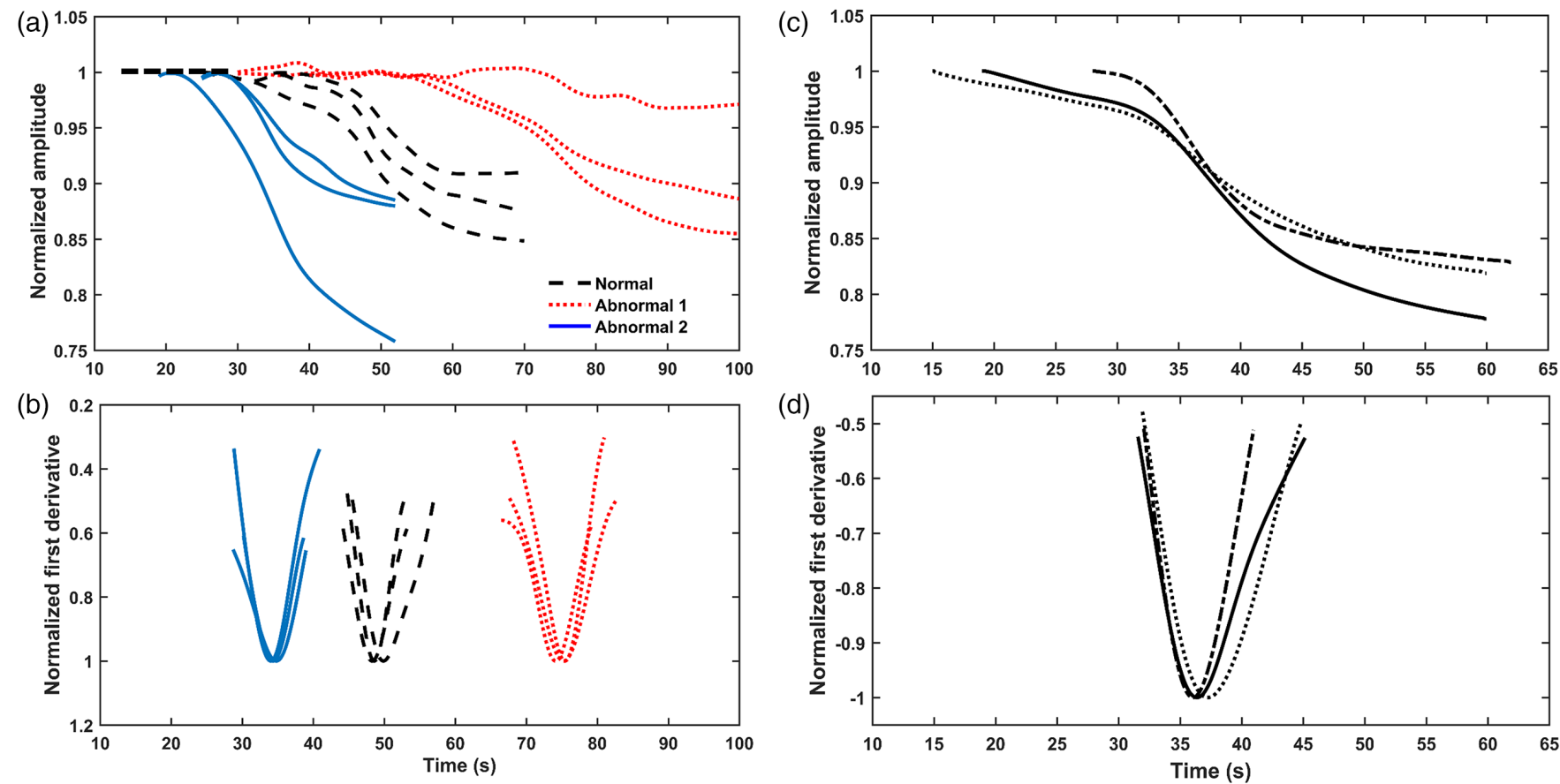

Fig. 5 (a) aPTT tests with normal, abnormal 1, and abnormal 2 control plasmas. Each test was repeated three times with the same control plasma samples but different cartridges. (b) First derivatives of control plasma results show the local minima. (c) aPTT tests were repeated for human whole blood sample three times with the same sample but different cartridges. (d) First derivatives of whole blood results show the local minima. $\sigma=0.76 \mathrm{~s}$ and $\mathrm{RSD} \%=2$.

Table 1 Normalized aPTT test results of control plasmas versus manufacturer reference data. $\sigma$ is standard deviation and RSD\% is relative standard deviation.

\begin{tabular}{lccccccccc} 
& \multicolumn{3}{c}{ Fiber-based sensor } & & \multicolumn{4}{c}{ Datasheet } \\
\cline { 2 - 3 } & $\begin{array}{l}\text { Normalized } \\
\text { aPTT ratio }\end{array}$ & $\sigma$ & RSD\% & & $\begin{array}{c}\text { Normalized } \\
\text { aPTT ratio }\end{array}$ & $\sigma$ & RSD\% \\
\hline Normal & 1.03 & 0.014 & 1.36 & 1.03 & 0.007 & 0.68 \\
Abnormal 1 & 1.45 & 0.007 & 0.48 & & 1.4 & 0.015 & 1.05 \\
Abnormal 2 & 2.22 & 0.012 & 0.54 & & 2.2 & 0.038 & 1.73 \\
\hline
\end{tabular}

Our system was tested with whole blood samples collected from a 24-year-old male healthy donor. The donation of human whole blood samples from healthy donors for this study was approved by the Koç University Ethical Committee, and healthy volunteers signed an informed consent. Blood samples were stored in 1.8-mL citrated blood vials (Greiner Bio One Vacuette 454326 polyethylene terephthalate sodium citrate blood collection coagulation tube, $3.2 \%$ sodium citrate). Before measurements, all blood samples, reagents, and the system were warmed to $37^{\circ} \mathrm{C}$. For aPTT tests, $50 \mu \mathrm{L}$ of whole blood was mixed with $50-\mu \mathrm{L}$ micronized silica/platelet substitute mixture (DIAGEN, UK, lot No.: SP66). Citrated vials bind the $\mathrm{Ca}^{+}$ions present in the whole blood samples. It is important to add $\mathrm{Ca}^{+}$ ions since $\mathrm{Ca}^{+}$ions aid the fibrin formation. The whole bloodreagent mixture was incubated for exactly $5 \mathrm{~min}$. After incubation, $50 \mu \mathrm{L}$ of $25-\mathrm{mM} \mathrm{CaCl}_{2}$ solution was mixed with the whole blood-reagent mixture, and $12 \mu \mathrm{L}$ of this final mixture was inserted into the cartridge immediately. Since whole blood samples are citrated, the coagulation process does not start until the introduction of $\mathrm{CaCl}_{2}$. Therefore, mixing of $\mathrm{CaCl}_{2}$ solution into the mixture is taken as $t=0$. While coagulation was observed through amplitude, the remaining sample was pipetted regularly and observed for clot forming to have an external confirmation for coagulation.

Figure 5(c) shows aPTT measurements of the blood samples taken from the same donor. Similar to control plasma tests, a sharp decrease in amplitude is observed when coagulation occurs. For three different tests, coagulation times were calculated at 36, 36.5, and $37.5 \mathrm{~s}$ using first derivatives [Fig. 5(d)]. Since there is no control whole blood sample readily available for coagulometer calibration, control plasma measurements were used for calibration purposes. Test results of whole blood samples yielded $0.76 \mathrm{~s}$ standard deviation and 2\% RSD, encouraging a PoC coagulameter application for the proposed system.

\section{Conclusions}

In this study, a direct mechanical coagulation measurement platform enabling measurements from whole blood samples was demonstrated. The system consists of a reader unit and a disposable cartridge with a simple fabrication process. Disposable cartridges have fiber optic-based sensors and microfluidic channels. The readout was performed optically, which is enabled by the microfluidic design. Since the optical readout is performed by a single photodetector, the cartridge and optical interface of the reader unit are simple compared with other optical readout architectures where laser Doppler vibrometer ${ }^{22}$ or diffractive optics ${ }^{23}$ were used. 
The fluid stop region is an important invention in our design. It was added to the microfluidic channel to prevent fluids from interfering with the optical readout and enabled high SNR optical signals.

aPTT tests were performed successfully with various control plasmas and with whole blood samples with RSD\% less than $2 \%$ in all cases. Repeatability of the results and the precision in coagulation time measurement were very good. Results reported in this study were obtained from a bench top prototype; a handheld reader unit prototype is currently under development. Initial results and simplicity of the cartridge design promise a low-cost PoC device for blood coagulation measurement.

\section{Disclosures}

The authors have no relevant financial interests in this article and no potential conflicts of interest to disclose.

\section{Acknowledgments}

This work was supported by the TÜBITAK 113 S074 grant. We thank Dr. Onur Cakmak, Dr. Aref Mostafazadeh, and Erhan Ermek for their help with the experimental procedures.

\section{References}

1. W. Korte et al., "Preoperative fibrin monomer measurement allows risk stratification for high intraoperative blood loss in elective surgery," Thromb. Haemostasis 94(1), 211-215 (2005).

2. V. L. Ng, "Liver disease, coagulation testing, and hemostasis," Clin. Lab. Med. 29(2), 265-282 (2009).

3. S.-W. Lin et al., "Dengue virus nonstructural protein $\mathrm{NS}_{1}$ binds to prothrombin/thrombin and inhibits prothrombin activation," $J$. Infect. 64(3), 325-334 (2012).

4. S. Lison and M. Spannagl, "Monitoring of direct anticoagulants," Wien. Med. Wochenschr. 161(3-4), 58-62 (2011).

5. J. W. Ojito et al., "Comparison of point-of-care activated clotting time systems utilized in a single pediatric institution," J. Extra Corpor. Technol. 44(1), 15-20 (2012).

6. C. M. Jackson and M. P. Esnouf, "Has the time arrived to replace the quick prothrombin time test for monitoring oral anticoagulant therapy?" Clin. Chem. 51(3), 483-485 (2005).

7. L. F. Harris, V. Castro-López, and A. J. Killard, "Coagulation monitoring devices: past, present, and future at the point of care," TrAC Trends Anal. Chem. 50, 85-95 (2013).

8. M. T. Ganter and C. K. Hofer, "Coagulation monitoring: current techniques and clinical use of viscoelastic point-of-care coagulation devices," Anesth. Analg. 106(5), 1366-1375 (2008).

9. D. Pacaud et al., "Assessment of blood volumes obtained by capillary punctures in older children and adolescents with diabetes. $\dagger 558$, Pediatr. Res. 39(S4), 95-95 (1996).

10. C. C. Shih et al., "Quantitative evaluation of thrombosis by electrochemical methodology," Thromb. Res. 111(1-2), 103-109 (2003).

11. S. Zhang, G. Wright, and Y. Yang, "Materials and techniques for electrochemical biosensor design and construction," Biosens. Bioelectron. 15(5-6), 273-282 (2000).

12. D. Barcellona et al., "Point-of-care (POCT) prothrombin time monitors: is a periodical control of their performance useful?" Thromb. Res. 123(5), 775-779 (2009).

13. CoaguChek XS, Evaluation Report 06024, Roche, Germany, http://www. roche.co.uk/content/dam/internet/corporate/roche/en_GB/documents/ Evaluation_Report_-_CoaguChek_XS.pdf (09 February 2017).

14. C. L. Yang et al., "Design and evaluation of a portable optical-based biosensor for testing whole blood prothrombin time," Talanta 116, 704-711 (2013)

15. M. M. Tripathi et al., "Assessing blood coagulation status with laser speckle rheology," Biomed. Opt. Express 5(3), 817 (2014).

16. H. Li et al., "Blood coagulation screening using a paper-based microfluidic lateral flow device," Lab Chip 14(20), 4035-4041 (2014).
17. A. M. H. P. Van Den Besselaar et al., "Analytical accuracy and precision of two novel point-of-care systems for INR determination," Thromb. Res. 135(3), 526-531 (2015).

18. E. C. Mpock, B. Clawson, and S. P. Murphy, "System for performing blood coagulation assays and measuring blood clotting times," misc, Google Patents (2007).

19. A. Dogariu, "Optical coagulation monitor and method of use," misc, Google Patents (2010).

20. B. Gregor, R. Bansil, and J. D. Spring, "Optical blood coagulation monitor and method," misc, Google Patents (2014).

21. O. Cakmak et al., "MEMS based blood plasma viscosity sensor without electrical connections," in IEEE Sensors (2013).

22. O. Cakmak et al., "A cartridge based sensor array platform for multiple coagulation measurements from plasma," Lab Chip 15(1), 113-120 (2015).

23. O. Cakmak et al., "Microcantilever based disposable viscosity sensor for serum and blood plasma measurements," Methods 63(3), 225232 (2013).

24. S. Z. Lulec et al., "Simultaneous self-sustained actuation and parallel readout with MEMS cantilever sensor array," in IEEE 25th Int. Conf. on Micro Electro Mechanical Systems (MEMS), pp. 644-647 (2012).

25. A. Mostafazadeh, G. G. Yaralioglu, and H. Urey, "Optical fiber array based simultaneous parallel monitoring of resonant cantilever sensors in liquid," Sens. Actuators A 242, 132-139 (2016).

26. A. S. Wolberg et al., "A systematic evaluation of the effect of temperature on coagulation enzyme activity and platelet function," J. Trauma Inj. Infect. Crit. Care 56(6), 1221-1228 (2004).

27. P. Evans, K. Hawkins, and P. Williams, "Rheometry for blood coagulation studies," Rheol. Rev. 2006, 255-291 (2006).

28. J. W. M. Chon, P. Mulvaney, and J. E. Sader, "Experimental validation of theoretical models for the frequency response of atomic force microscope cantilever beams immersed in fluids," J. Appl. Phys. 87(8), 39783988 (2000).

29. C. A. Van Eysden and J. E. Sader, "Frequency response of cantilever beams immersed in viscous fluids with applications to the atomic force microscope: arbitrary mode order," J. Appl. Phys. 101,044908 (2007).

30. J.-L. Gennisson, S. Lerouge, and G. Cloutier, "Assessment by transient elastography of the viscoelastic properties of blood during clotting," Ultrasound Med. Biol. 32(10), 1529-1537 (2006).

31. A. N. Yaroslavsky et al., "Optical properties of blood in the near-infrared spectral range," Proc. SPIE 2678, 314-324 (1996).

32. C. P. M. Hayward et al., "Approaches to investigating common bleeding disorders: an evaluation of North American coagulation laboratory practices," Am. J. Hematol. 87(Suppl. 1), S45-S50 (2012).

33. United States Medical Licensing Examination Clinical Knowledge, USMLE, http://www.usmle.org/pdfs/step-2-ck/2017_Step2CK_Sample Items.pdf (30 October 2017).

34. P. Thiagarajan, V. Pengo, and S. S. Shapiro, "The use of the dilute Russell viper venom time for the diagnosis of lupus anticoagulants," Blood 68(4), 869-874 (1986).

35. S. Yuan, C. Ferrell, and W. L. Chandler, "Comparing the prothrombin time INR versus the APTT to evaluate the coagulopathy of acute trauma," Thromb. Res. 120(1), 29-37 (2007).

Yusuf Samet Yaraş received his bachelor's of science degree from Sabanci University in electronics engineering. In July 2013, as a MSc student, he joined the Optical Microsystems Laboratory. Currently, he is working as a research assistant under the supervision of Prof. HakanÜrey at the OML and as a teaching assistant at Koç University.

Ali Bars Gündüz received his BS degree from Koç University, Physics Department in 2015. He joined the OML as a research engineer in 2015, and he is working on biosensor project.

Gökhan Sağlam received his BSc and MSc degrees in biomedical engineering. He worked in medical sector for eight years as a product manager and sales and marketing manager. He works as an coordinator in coagulation management device commercialization and $R \& D$ project.

Selim Ölçer is an electronics technician, working as a laboratory coordinator in Engineering Department. He has a 20 years of experience 
on embedded systems, analog-digital electronics design and software development.

Fehmi Civitçi is graduated from Electrical and Electronics Engineering Department, METU and stayed to work there for four years as a research assistant. His topic was uncooled infrared detector pixels. Then, he finished his $\mathrm{PhD}$ in Twente University working on hybrid integration of optical waveguides and photodetectors. He also developed a prism based integrated optical spectrometer. As a postdoc, he worked in KOÇ University on fiber opticsensors.

Ibrahim Baris graduated from the Department of Molecular Biology and Genetics at Bogazici University in 2000 . He received his PhD in 2008 from the same department. He joined the lab of Prof. HalilKavaklı (Koc University, Department of Molecular Biology and Genetics) as a research fellow in 2008 . He received associate prof. title in 2013. He is interested in molecular basis of disease and development of genetic testing assays.
Göksenin Yaralioğlu received his $\mathrm{PhD}$ from Electrical and Electronics Engineering Department, Bilkent University on the noise analysis of atomic force microscope. After that he worked as an engineering research associate at Stanford University. He worked on MEMS, ultrasound, material characterization and atomic force microscopy. Later he directed test activities to calibrate MEMS sensors in a private company. He published his research in international journals and in book chapters. He holds seven patents.

Hakan Urey is a professor of electrical engineering at Koç University, Istanbul. He received BS degree from METU, Ankara and MS and $\mathrm{PhD}$ degrees from Georgia Tech all in electrical engineering. He worked for Microvision before joining Koç University. He is the inventor of nearly 50 issued and pending patents about displays, MEMS, optical sensors, and microtechnologies. He published about 200 papers in international journals and conferences and received ERC Advanced Grant in 2013. 